\title{
NeuroGlasses: A Neural Sensing Healthcare System for 3-D Vision Technology
}

\author{
Fang Gong, Student Member, IEEE, Wenyao Xu, Student Member, IEEE, Jueh-Yu Lee, Student Member, IEEE, \\ Lei He, Senior Member, IEEE, and Majid Sarrafzadeh, Fellow, IEEE
}

\begin{abstract}
D vision technologies are extensively used in a wide variety of applications. Particularly glasses-based 3-D technology facilities are increasingly becoming affordable to our daily lives. Considering health issues raised by 3-D video technologies, to the best of our knowledge, most of the pilot studies are practiced in a highly-controlled laboratory environment only. In this paper, we present NeuroGlasses, a nonintrusive wearable physiological signal monitoring system to facilitate health analysis and diagnosis of 3-D video watchers. The NeuroGlasses system acquires healthrelated signals by physiological sensors and provides feedbacks of health-related features. Moreover, the NeuroGlasses system employs signal-specific reconstruction and feature extraction to compensate the distortion of signals caused by variation of the placement of the sensors. We also propose a server-based NeuroGlasses infrastructure where physiological features can be extracted for real-time response or collected on the server side for long term analysis and diagnosis. Through an on-campus pilot study, the experimental results show that NeuroGlasses system can effectively provide physiological information for healthcare purpose. Furthermore, it approves that 3 -D vision technology has a significant impact on the physiological signals, such as EEG, which potentially leads to neural diseases.
\end{abstract}

Index Terms-Healthcare system, patten recognition, signal processing.

\section{INTRODUCTION}

$\mathbf{S}$ INCE David Brewer invented "Stereoscope" in 1844, which is the first device taking 3-D photographic picture, there has been huge amount of researches and developments in 3-D vision. Existing stereoscopic technology [1] includes 3-D glasses, augmented reality, autostereograms, lenticular prints, Piku-Piku and much more. Eventually the only commercialized technology is 3-D glasses due to reliability, stability, and cost. Nowadays 3-D glasses has become a popular and indispensable element in consumer electronics and entertainment. On January 31, 2010, James Cameron's 3-D movie "Avatar" became the first film to earn more than 2 billion. On April 3, 2010, Sky launched the first 3-D broadcasts in U.K., and 3-D televisions (with glasses) are out-of-stock in all the markets in London. These days all the giant TV manufacturers jump into the competition of this hot

Manuscript received March 22, 2011; revised September 6, 2011; accepted November 6, 2011. Date of publication November 18, 2011; date of current version March 7, 2012.

F. Gong, W. Xu, J.-Y. Lee, and L. He are with the Department of Electrical Engineering, University of California, Los Angeles, CA 90095 USA (e-mail: fang08@ee.ucla.edu;wxu@ee.ucla.edu; royjylee@ucla.edu; lhe@ee.ucla.edu).

M. Sarrafzadeh is with the Department of Computer Science, University of California, Los Angeles, CA 90095 USA (e-mail: majid@cs.ucla.edu).

Color versions of one or more of the figures in this paper are available online at http://ieeexplore.ieee.org.

Digital Object Identifier 10.1109/TITB.2011.2176539 market, and the 3-D television set becomes affordable to most of the family.

However, as every emerging technology is criticized in its early stage, 3-D technology has to face the similar situation. Unlike some products struggle with the marketing or pollution, health and safety issues, including physical and mental strain, accompany 3-D technology with its birth. One of the major TV manufacturers "Samsung" issued a warning about possible health effects with the formal product release. It describes a long list of hazards potentially associated with technology and mentions a number of strict use rules. Actually, the side-effects are far beyond people's expectation. A lot of viewers complained about dizziness in front of 3-D TV or movies for a slightly long time. Moreover, the report from one Taiwan public media said that one cinemagoer with chronic high blood pressure died from sudden death when watching "Avatar."

Now it is time for us to pay more attention on corresponding health researches. However, the investigation of these possible effects is difficult. No watcher accepts to suffer a medical examination before they enjoy the visual feast; spreading inspective health meters on the watcher's body is not a wise idea, in the sense that, it will diminish the users' entertainment. Accordingly, to take a proper action, a comprehensive and noninvasive sensing technology is needed.

Wireless health technology is a feasible way to address this issue. It is rooted in the World Health Organization established project for the environment in 1996. With the popular health concern continually growing in the past decade, wireless health becomes a new undependable branch both in industry and academia. Different from traditional human computer interface [2], wireless health technology has an invisible interface and play context-aware computing in the background.

Our research sought to develop a system to provide wireless health monitoring for 3-D video watcher. The service can be provided in daily life outside of traditional highly-controlled scientific laboratories. In this paper, we presented the design, implementation and evaluation of NeuroGlasses, and a mobile healthcare monitoring system. This system could provide a noninvasive realtime monitor and feedback of physiological status for 3-D video watchers. Furthermore, we proposed a signalspecific physiological signal process algorithm to improve SNR to get more accurate features. There are two main contributions of this paper: the infrastructure of the monitoring system and signal-specific reconstruction algorithm.

The structure of the remaining part is as follows. In Section II, we discuss 3-D side-effects and related physiological features. The system architecture and implementation are introduced in 
TABLE I

COMPARISON OF CHARACTERISTICS OF THE PHYSIOLOGICAL SignalS

\begin{tabular}{|c|c|c|c|c|c|}
\hline & Type & Origin & Frequency & Location & Pathology \\
\hline & ECG & Heart & $1-40 \mathrm{~Hz}$ & arm, leg, head, chest & inverted $T$ waves, hyperacute $T$ waves \\
\hline & EOG & Eye & $1-20 \mathrm{~Hz}$ & head & ophthalmology \\
\hline & EMG & Muscle & $7-20 \mathrm{~Hz}$ & arm, leg, head, chest & neuromuscular diseases \\
\hline \multirow{3}{*}{ EEG } & Theta Waves & \multirow{3}{*}{ Brain } & $4-7 \mathrm{~Hz}$ & \multirow{3}{*}{ head } & memory tasks \\
\hline & Alpha Waves & & $8-12 \mathrm{~Hz}$ & & sleep disorder \\
\hline & Beta Waves & & $12-30 \mathrm{~Hz}$ & & Parkinson's disease \\
\hline
\end{tabular}

Section III. Afterwards, physiological signal processing algorithm is described in Section IV. We presented the experimentation in Section V. Conclusion and future work are summarized in Section VI.

\section{BACKGROUND}

\section{A. Physiological Signals}

Physiological signals denote chemical and electrical signals generated by the tissue activity inside human bodies, which can provide some clues about vital health signs of human beings. For example, EEG is emitted by the brain, and EMG is emitted by the muscles. Normally, the physiological signal can be measured by the biopotential electrodes, enabling the people to explore and explain the biological problems with electronic technology. Here we listed the characteristics of classical physiological signals in Table I. Each signal originates from the specific organs and is linked to the featured disease. According to the signal distribution, the head is the largest concentration of the physiological signals required for our research. Additionally, some physiological signals overlap with each other, and the same frequency interference will be a practical challenge.

\section{B. Related Work}

Pervasive sensing [3]-[6] is a popular research topic in the last decade. Currently, deployment of biopotential or inertial sensors attached on carry-on devices for health applications [7]-[12] receives more attention. However, there are only limited study on the safety evaluation of 3-D vision technology. Scientists from University of California Berkeley [13] built a stereo machine to investigate the unhealthiness. They claim that the physical strain, such as eye fatigue, root in abnormal eye movements; researchers at University of Central Florida [14] claim unknown disparities will cause mental strain, vertigo, and nausea. Recently, Entertainment Technology Center [15] at the University of Southern California began to collect the users' experience in order to dig out the pathogen and improve 3-D technology. All these afore-mentioned works require high-controlled environments, therefore, their needed professional assistance and device cost are very expensive. To address this problem, portable and low-cost physiological signal monitoring platforms are on demands.

\section{NeUROGLASSES SYSTEM}

\section{A. System Architecture Overview}

Fig. 1 shows the system architecture. Here we use an application scenario to explain the flow of the data acquisition,
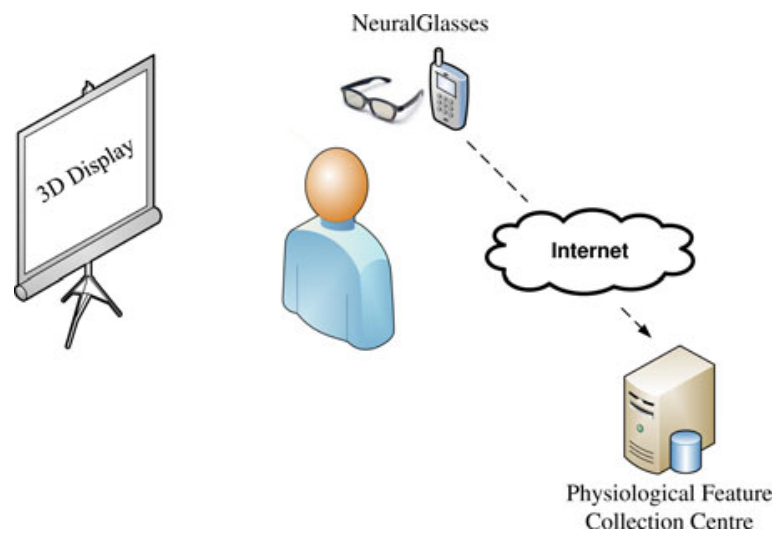

Fig. 1. Overview of NeuroGlasses system architecture.

data transmission, data mining, data archiving, and data management in the NeuroGlasses system. In this case, a watcher with NeuroGlasses sits in front of 3-D display. While he or she watches the movies, the neural sensors on NeuroGlasses noninvasively collect his/her physiological data and send them to the smart phone device through the near-field body channels. Meanwhile, smart phone will also upload these received data to remote servers. The health-related computing could be launched on either mobile device or remote server.

\section{B. Hardware}

1) Neural Sensor Sets: Based on the analysis in Section II, the NeuroGlasses system should be capable of sensing different kinds of physiological signals that characterize the health status. We do not take research-grade neural sensor sets into account. It is because that deploying research-grade neural acquisition system needs professional assistance. The sensors have to be put in the accurate location. Moreover, some of them, such as EEG electrodes, need conductive cream, which is unacceptable in daily use. So we vote for cheaper sensor solutions although the interference will make the signal processing more challenging. Some cheaper neural headsets are available in the form of off-the-shelf products. Table II shows a comparison of selected popular neural headsets, where "-" means the item is unknown. NeuroSky cannot measure EOG and ECG signal; Emotiv EPOC is a powerful EEG headset and performs a comprehensive sensing. However, its driver is difficult to use. Campbell et al. [16] use this headset, and they have to use a laptop for relaying the signal between smartphone and sensor, which is not suitable in our application scenario. Therefore, we choose OCZ NIA as the front-end sensor. NIA is a headband with one neurosensor. Whenever the user wears it, the sensor will be attached to the 
TABLE II

COMPARISON AMONG OFF-THE-SHELF NeURAL HEADSETS

\begin{tabular}{|c|c|c|c|}
\hline Properties & Mindset[17] & Emotiv[18] & NIA [19] \\
\hline Sensor Numbers & 5 & 16 & 1 \\
Sensor Weight & $165 \mathrm{~g}$ & $200 \mathrm{~g}$ & $125 \mathrm{~g}$ \\
Retail Price & $\$ 199$ & $\$ 299$ & $\$ 70$ \\
System requirements & Low & High & Low \\
EMG measurability & Yes & Yes & - \\
EOG measurability & No & Yes & Yes \\
ECG measurability & No & - & - \\
EEG measurability & Yes & Yes & Yes \\
\hline
\end{tabular}

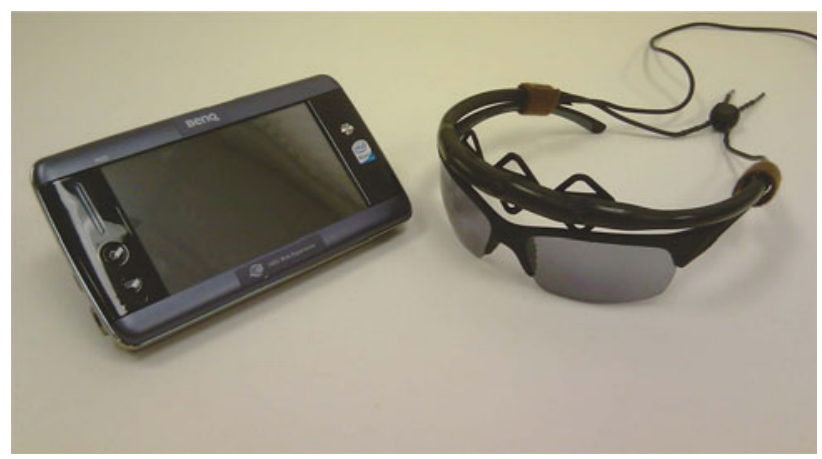

Fig. 2. NeuroGlasses system consisting of PC phone and sensors.

skin at the forehead part. Different from typical two-pin neurosensor, NIA has three electrodes; the middle one is the the ground pin, and the side ones measure the differential signal value. The sensing signal is amplified with gain of 50. After filtering, it is quantified with 24-bit ADC, and sampling rate is $3.90625 \mathrm{KHz}$.

2) System Setup: We use PC phone BenQ S6 with $3 \mathrm{G}$ and WiFi as the data acquisition device and central control unit. The entire system is shown in Fig. 2. The PC phone receives the signal from the sensor and performs some customized lightweight computation, displays the results, and provides the feedback. Then, the raw data or processed data are uploaded to the remote server through $3 \mathrm{G}$ or $\mathrm{WiFi}$ wireless connection. On the server side, a comprehensive data mining procedure is conducted for feature analysis.

\section{Software Architecture}

NIA sensor receives the comprehensive physiological data and transferred them to PC phone. The software architecture consists of three layers which are shown in Fig. 3: the sensor layer, the signal layer, and the application layer. The sensor layer abstracts from the low-level details and generalizes the sensor API; The signal layer uses the cognition algorithm of retrieving the feature of the physiological signals. Based on the analytic results from the recognition layer, the application layer makes an analysis, performs the feedback, and archives the data.

1) Sensor Layer: The software of the sensor layer is implemented in $\mathrm{C}++$. In this layer, several physical-level data processing are performed. The driver here has two main functions: flow control and error detection. With 24-bit ADC and $3.90625 \mathrm{KHz}$ sampling frequency, the data rate from the sensor is $93750 \mathrm{bps}$.

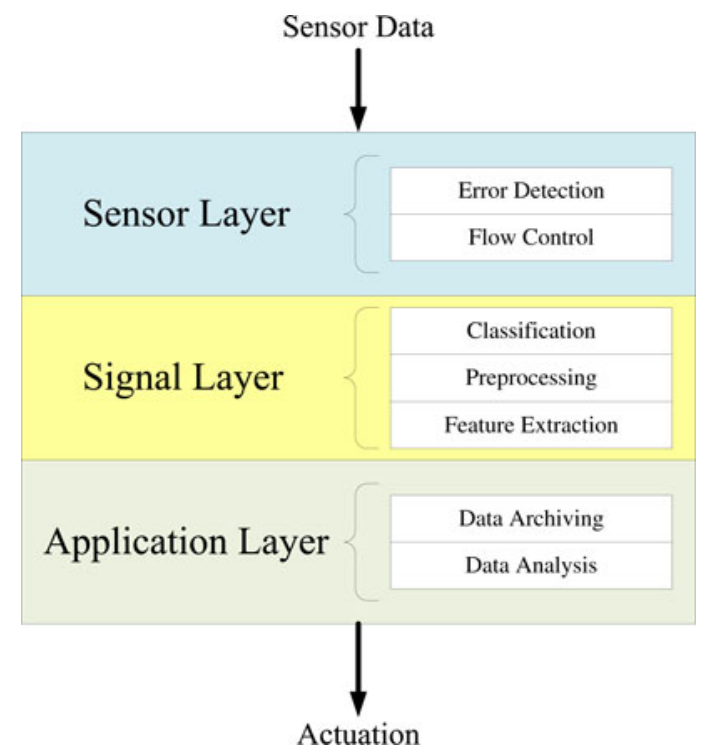

Fig. 3. Software architecture.

In term of realtime consideration and computation limitation, the driver empties the buffer every 10 samples.

2) Signal Layer: The signal layer is built on the sensor layer and extract the features of the biosignal. As shown in Fig. 3, it has three phases: classification, preprocessing, and feature extraction.

a) Classification: The phase "Classification" separates the different kinds of physiological signals from the comprehensive data. Filter design is the main content in this phase. Due to the complexity of the filter, the tradeoff between the quality and the computation cost is the key issue in this phase.

b) Preprocessing: The phase "Preprocessing" is a contextaware algorithm for the information loss of the data. It reduces the interference based after the classification. The lightweight action is performed in the mobile device BenQ S6, and the server runs the computationally expensive task.

c) Feature Extraction: In this phase, we extract the features describing the characteristics of the physiological signals. The extraction algorithm is dependent on the mark of the features in the time domain or the frequency domain.

3) Application Layer: The application layer is implemented on both PCphone and server. The partitioning depends on the requirement of the task. Basically, there are two kinds of action here. One is the data storage, and it is implemented in servers. The other is the signal analysis. If the criteria of analysis is realtime, the computation should be executed on the phone side; if the key factor is accuracy or long-run trend, server will take over the task.

\section{Physiological Signal Processing}

In this section, we introduce the structure of physiological signal processing. Fig. 4 shows the process of data flow in the NeuroGlasses system. In our system, physiological signal processing consists of filtering and data reconstruction. 


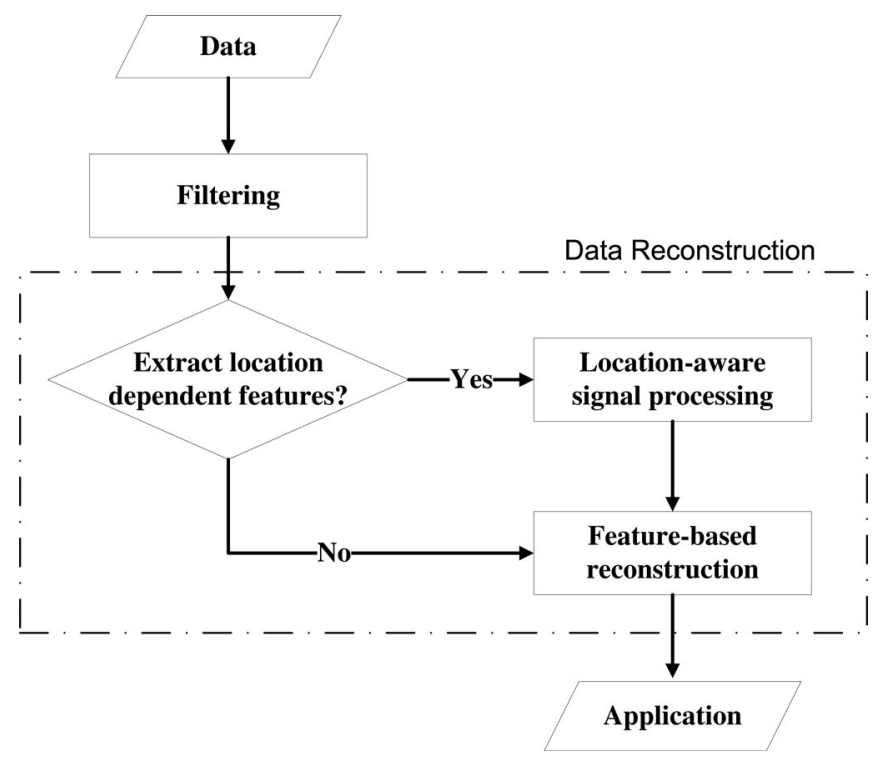

Fig. 4. Physiological signal processing flow.

\section{A. Filtering}

1) Filter Design: The raw data from the sensor layer has a low signal to noise ratio (SNR) and it is necessary to improve SNR by filtering before further analysis. There are two basic filter structures: Finite impulse response (FIR) filter and infinite impulse response (IIR) filter. Under the same pass-band requirement, IIR is low cost and requires less computation in the filter realization. However, IIR is nonlinear and will distort the signal, which will weaken or even remove the features of physiological signals. Thus, we choose FIR filter in our implementation. The filter design for each physiological signal is referred to Table I.

In fact, the behaviors of the filter are not ideal, such as the gainto-attenuation band is significant; the in-band gain is not smooth. These nonideal characteristics will introduce interference and make the feature extraction challenging.

2) Interference Analysis: Basically there are three types of unremovable interference in the filtered signal:

a) Co-Channel Noise (CCN): $\mathrm{CCN}$ is the noise with the same frequency band of the desired signal. This kind of noise cannot be removed even with ideal filters.

b) Adjacent Channel Noise ( $A C N)$ : The gain-to-attenuation throughout in the real filter is not zero. The noise in this transition zone will not be attenuated completely, which will affect SNR.

c) In-Band Distortion (IBD): Different from CCN, IBD is generated by that the signal within the pass-band will become distorted because the gain is not uniform.

Fig. 5 shows the signal distortion after filtering. It is obvious that there is still significant fluctuation coupling with the signal wave. Moreover, the interference with large magnitude will change the profile of the signal. Therefore, we need a signalspecific methodology to restore the physiological features.

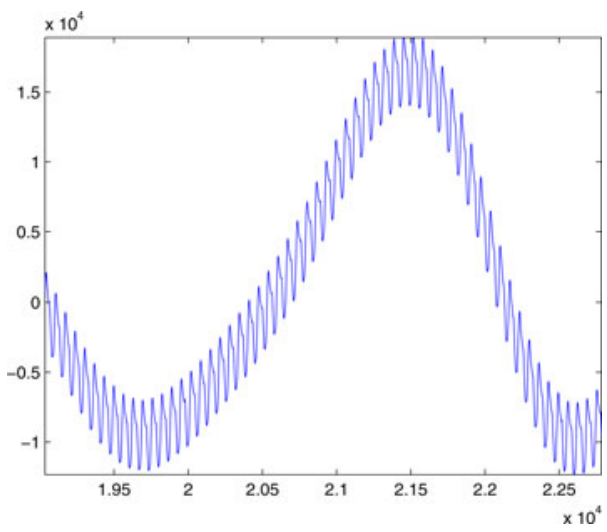

Fig. 5. Noise in neural signals ( $\mathrm{x}$-axis is time, $\mathrm{y}$-axis is magnitude).

\section{B. Data Reconstruction}

As shown in the algorithm flow in Fig. 4, the filtered data needs to be reconstructed before feature extraction due to significant signal distortion.

1) Location-Aware Correction: The sensor location depends on how the user wears NeuroGlasses individually. The variation of the sensor location greatly affects the sensing data. In reality, NeuroGlasses restrict the position of the sensor and the location variation will be within some range. For simplicity, we model that there are three different locations on the forehead: left, center, right.

Specific signal processing tools, such as Principal Component Analysis [20] and Local Discriminant Analysis [21], can explore more accurate location-dependent features. Considering the whole algorithm implementing in the carry-on device, we employ featured-based reconstruction to correct other distortions.

2) Feature-Based Reconstruction: In order to extract the physiological features accurately, we propose a moving-window algorithm to detect and further correct the signal distortions. Our algorithm has been outlined as under:

With the filtered physiological signal as input, our algorithm can build a moving-window and move it along the time axis. In this process, we define an segmentation factor as $s_{\gamma}=\partial f(t) / \partial t$ to detect the noise interference, where $f(t)$ is the amplitude of physiological signal under studied. Also, $s_{\gamma}^{k}$ can be approximated with $\left(f_{k}-f_{k-1}\right) / \Delta t$ at each time-step. When the segmentation factor reaches certain threshold value, one concave can be identified and thus two spikes can be separated.

Next, we can reconstruct the shape of distortion-free physiological signal according to different interference impact; the noise peak should be removed for positive interference impact, while all the peaks within the moving-window should be combined for negative interference impact. As such, the interference can be eliminated from the physiological signals and the accuracy of feature extraction can be improved significantly.

\section{EVALUATION}

To verify the correctness and effectiveness of our proposed algorithm, we run an on-campus pilot with 20 subjects, including 


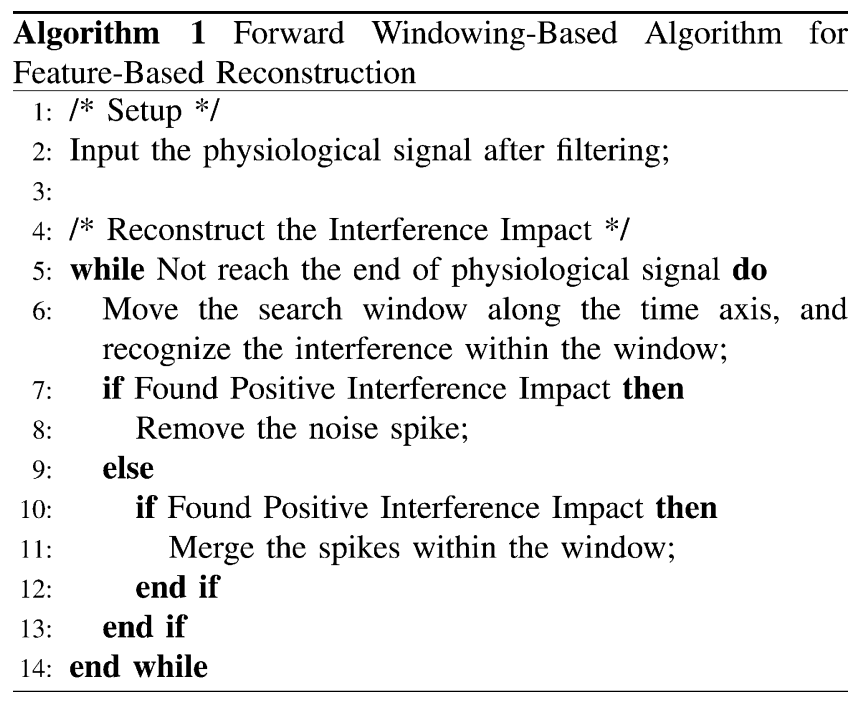

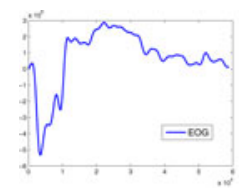

(a)

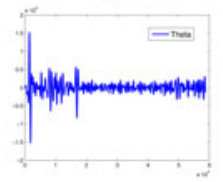

(d)

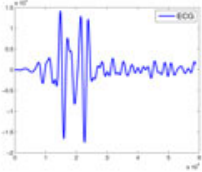

(b)

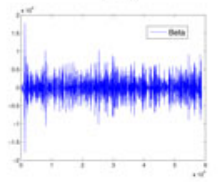

(e)

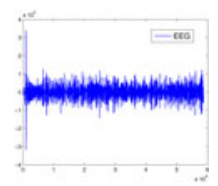

(c)

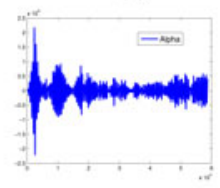

(f)
Fig. 6. Context-aware classification for raw data (x-axis is time, $y$-axis is magnitude). (a) EOG. (b) ECG. (c) EEG. (d) Theta waves. (e) Beta waves. (f) Alpha waves.

10 male volunteers and 10 female volunteers. There are two sets of experiments. The first one is to examine the performance of our algorithm, including location-aware correction, physiological feature extraction, etc. The second one is to validate the influence of 3-D technology from the change of the physiological features.

\section{A. Signal Reconstruction and Feature Extraction}

1) Experiment Setup: We implemented the algorithm described in Section IV. In the experiment, the measured objects watch 3-D videos when NeuroGlasses can record their physiological signal and perform the analysis. The experimental purpose is to examine whether NeuroGlasses could perform an accurate analysis in terms of physiological features.

2) Physiological Signal Classification: We collect a 16-s clip of the field data when the objects watch 3-D videos, which contain different physiological signals. To analyze these signals, the first step is to remove the noise and increase SNR. Then, we will do signal classification to analyze the physiological spectrum, including EOG, ECG, and EEG signals (including alpha, beta, theta waves) as shown in Fig. 6. The processing result show that our algorithm could effectively classify different physiological signals from raw sensing data.

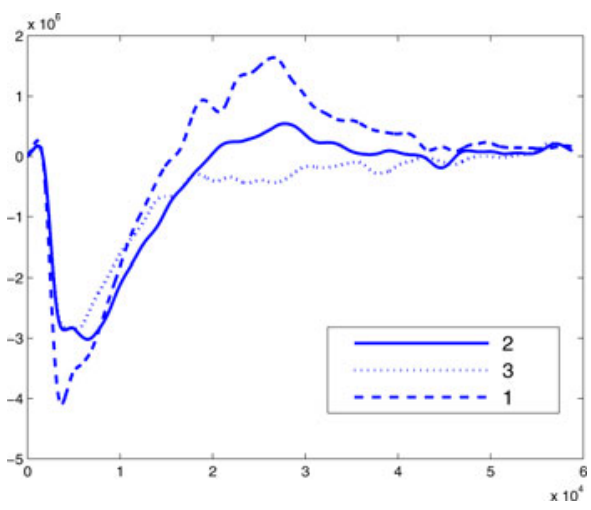

Fig. 7. EOG Location-aware correction (x-axis is time, $y$-axis is magnitude).

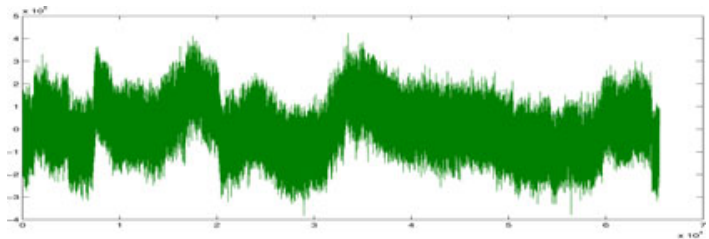

(a)

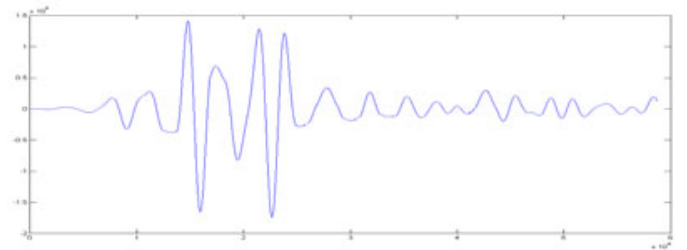

(b)

Fig. 8. Signal processing on ECG signal ( $\mathrm{x}$-axis is time, $\mathrm{y}$-axis is magnitude). (a) ECG signal without filtering. (b) Reconstructed ECG signal after filtering.

3) Signal Correction and Reconstruction: We further test the location-correction algorithm on EOG signals, as illustrated in Fig. 7, where Position 2 is the correct senor location (i.e., center of forehead). The remaining two positions denotes left and right location, respectively. It can be observed that our algorithm can efficiently eliminate the distortion due to different locations.

4) Physiological Feature Extraction: We also examined our algorithm in terms of physiological feature extraction. We use ECG as an example which is one of the three most important vital signs at a clinic and its significant features are ECG heart rate. In fact, ECG heart rate is the number of the heart beat per minute (hpm) and can vary dramatically according to the different physical states, such as exercise, sitting, and sleeping. Also it will be affected by mood, such as anger, happiness, and peace. It has been extensively used in clinical study for the basic guideline of the health status.

As shown in Fig. 8(a), ECG signal is interfered by the noise and heart rate cannot be calculated directly, which calls for the data reconstruction operation. Ideally, the reconstruction operation should remove the undesired noises and remain the significant features in the signal. Fig. 8(b) illustrates the reconstructed ECG signal from our proposed algorithm. 


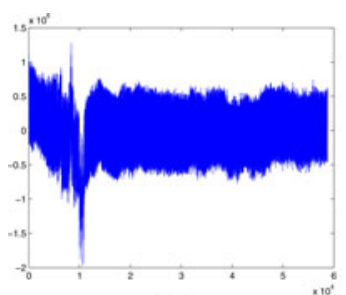

(a)

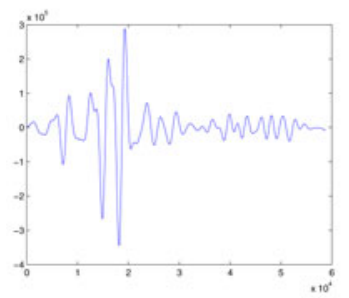

(b)

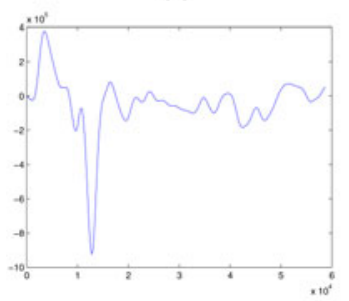

(e)

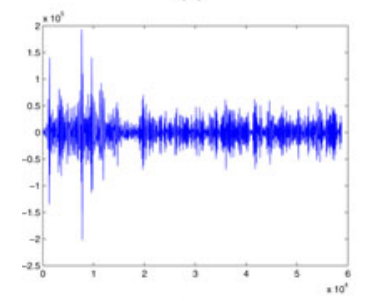

(g)

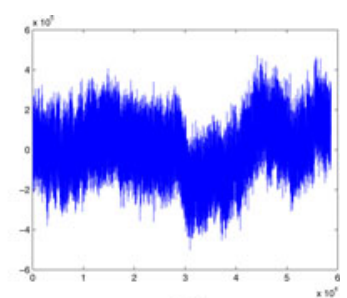

(b)

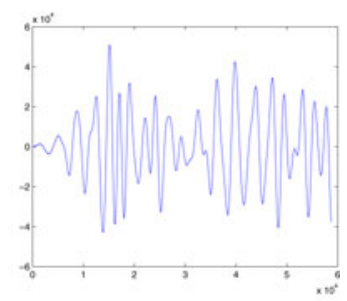

(d)

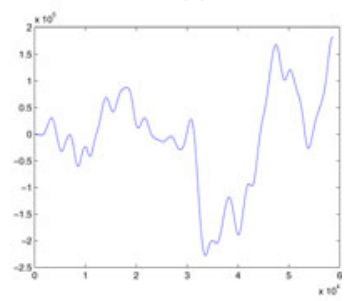

(f)

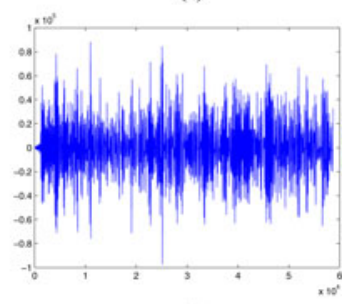

(h)
Fig. 9. Physiological Response: 2-D versus 3-D (x-axis is time, y-axis is magnitude). (a) 2-D raw data. (b) 3-D raw data. (c) 2-D ECG. (d) 3-D ECG. (e) 2-D EOG. (f) 3-D EOG. (g) 2-D beta waves. (h) 3-D beta waves.

In details, there are 18 pulses with $16 \mathrm{~s}$ so that we could calculate heart rate of this person around 67. In the same while, we used off-the-shelf heart rate meter to measure the ECG signal of the subjects. It shows that the calculated result from NeuroGlasses has the comparable accuracy with the ground truth and could offer reliable guidance for medical examination.

\section{B. Evaluation of 3-D Vision Impact on Subjects}

The second part is to investigate the 3-D technology influence on human beings. We performed a pilot test on campus; there were twenty objects comprising ten male and ten female. Each participant was required to put on the NeuroGlasses system and watch the same videos with different formats, including 2-D and 3 -D version. Note that these two videos had the same content and were displayed within the same environment, and the only difference was the vision technology. During the experiments, NeuroGlasses system recorded the neural signals. After that, the user experiments were to be surveyed as reference. For simplicity of presentation, the analyzed data from one of the subjects are illustrated in Fig. 9.

This case study demonstrates that the object shows evident abnormality on the physiological signal while watching the 3-D video. For example, it can be observed that 2-D raw data show small fluctuation and constant mean value along the time. In contrary, 3-D raw data have large variations (fluctuation) compared with the data in 2-D case, which means the brain status of the object is much more active within the 3-D case. It could be potentially associated with some neural disease. Accordingly, the extracted 3-D ECG signals have larger amplitude and more peaks. The similar phenomenon can be observed in other subjects.

\section{CONCLUSION}

In this paper, we propose NeuroGlasses and a physiological feature acquisition system for 3-D video goers. We also propose a lightweight location-aware signal correction and a featurebased reconstruction approach to compensate signal distortion due to the variation of sensor location or other noises. By a prototyping of NeuroGlasses system, we show that physiological features can be extracted and corrected effectively, where features can be processed on a mobile device, such as a PCphone, for online monitoring or stored on a server for long-term analysis through wireless internet connection. Furthermore, we show a significant difference on physiological features between 2-D video watchers and 3-D video watchers. In the future, a comprehensive study of physiological feature extractions that are related to health issues for 3-D technology will be involved. Then, we will develop an effective feature correction and extraction algorithm for these physiological features so that a more reliable health monitoring can be provided.

\section{REFERENCES}

[1] "Stereoscopy" Wikipedia. Available: http://en.wikipedia.org/wiki/ Stereoscopy

[2] "Human Computer Interaction," Wikipedia. Available: http://en. wikipedia.org/wiki/Human_computer_interaction

[3] Y. Hata, S. Kobashi, H. Yamaguchi, O. Ishikawa, N. Tsuchiya, and H. Nakajima, "Human health monitoring system of systems by non-contacted senors," in Proc. IEEE Int. Conf. Syst. Syst. Eng., Albuquerque, NM, 2009, pp. 1-5.

[4] K. Wu and X. Wu, "A wireless mobile monitoring system for home healthcare and community medical services," in Proc. 1 st Int. Conf. Bioinformat. Biomed. Eng., Wuhan, China, 2007, pp. 1190-1193.

[5] T. Matsukawa, T. Umetani, and K. Yokoyama, "Development of health monitoring system based on three-dimensional imaging using bio-signals and motion data," in Proc.29th Annu. Int. Conf. IEEE Eng. Med. Biol. Soc., Lyon, France, 2007, pp. 1523-1527.

[6] S. Lee, Y. Kim, G. Lee, B. Cho, and N. Lee, "A remote behavioral monitoring system for elders living alone," in Proc. Int. Conf. Control, Autom. Syst., Seoul, Korea, 2007, pp. 2725-2730.

[7] K. Lawrence, W. Wu, M. Batalin, T. Stathopoulos, and W. Kaiser, "Demonstration of active guidance with SmartCane," in Proc. 7th Int. Conf. Inf. Process. Sens. Netw., St. Louis, MO, 2008, pp. 537-538.

[8] R. Paradiso, G. Loriga, and N. Taccini, "A wearable health care system based on knitted integrated sensors," IEEE Trans. Inf. Technol. Biomed., vol. 9, no. 3, pp. 337-344, Sep. 2005.

[9] J. Smalls, Y. Wang, X. Li, Z. Chen, and K. Tang, "Health monitoring systems for massive emergency situations," in Proc. Syst., Appl. Technol. Conf., Farmingdale, NY, 2009, pp. 1-11. 
[10] C. Lin, M. Chiu, C. Hsiao, R. Lee, and Y. Tsai, "Wireless health care service system for elderly with dementia," IEEE Trans. Inf. Technol. Biomed., vol. 10, no. 4, pp. 696-704, Oct. 2006.

[11] S. Kim, D. Ryoo, and C. Bae, "U-healthcare system using smart headband," in Proc. Int. Conf. IEEE Eng. Med. Biol. Soc., Vancouver, BC, 2008, pp. 1557-1560.

[12] B. Masot, C. Gehin, R. Nocua, A. Dittmar, and E. McAdams, "A wearable, low-power, health-monitoring instrumentation based on a programmable system-on-chip," in Proc. Annu. Int. Conf. IEEE Eng. Med. Biol. Soc., Minneapolis, MN, 2009, pp. 4852-4855.

[13] T. Shibata, J. Kim, D. M. Hoffman, and M. S. Banks, "Visual discomfort with stereo displays: effects of viewing distance and direction of vergenceaccommodation conflict," Proc. SPIE: Stereoscopic Displays Appl., vol. 7863, pp. 1-9, Jan. 2001.

[14] [Online]. Available: http://www.creol.ucf.edu/Default.aspx

[15] P. Lelyveld, "Executive briefing: Basic visual perception concepts related to 3D Content," Entertainment Technology Center at USC (white paper), Jun. 2009. Available: http://www.etcenter.org/files/ public/090617_3D_primer.pdf

[16] A. Campbell, T. Choudhury, S. Hu, H. Lu, M. Mukerjee, M. Rabbi, and R. Raizada, "Neurophone: Brain-mobile phone interface using a wireless eeg headset," in Proc. 2nd ACM SIGCOMM Workshop Netw., Syst., Appl. Mobile Handhelds (MobiHeld' 10), New Delhi, India, 2010, pp. 3-8.

[17] NeuroSky Brain-Computer Interface Technologies, "Brain Wave Signal (EEG) of NeuroSky, Inc.," Dec. 15, 2009. Available: http://www.neurosky.com/AcademicPapers.aspx

[18] EmotiveSystems, "A new approach to mobile enabling enterprise applications," 2011. Available: http://www.emotive.com/

[19] Cyril Kowaliski, "OCZ's mind-control system examined," Mar. 20, 2007. Available: http://techreport.com/discussions/12070

[20] I. T. Jolliffe, Principal Component Analysis. New York: SpringerVerlag, Oct. 2002.

[21] R. Duda and P. Hart, Pattern Classification and Scene Analysis. Singapore: Wesley Publisher, 1973.

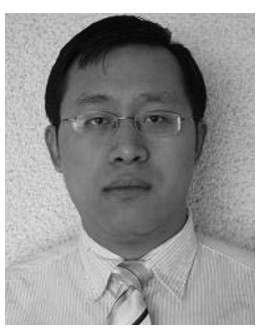

Fang Gong (S'08) received the B.S. degree in computer science from Computer Science Department at Beijing University of Aeronautics and Astronautics, China, in 2005, and the M.S. degree in computer science from Computer Science Department at Tsinghua University, China, in 2008. He is working toward the Ph.D. degree in the Electrical Engineering Department at University of California, Los Angeles.

His research interests mainly focus on numerical computing and stochastic techniques for computeraided design and wireless health applications.

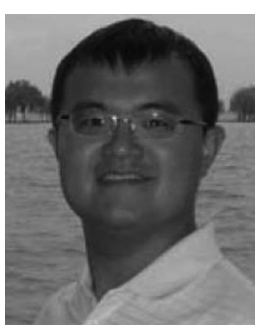

Wenyao Xu (S'08) received the B.S. and M.S. degrees in electrical engineering from Zhejiang University, Hangzhou, China, in 2006 and 2008, respectively. From 2008, He started his graduate study at the Department of Electrical Engineering and Computer Sciences of the University of California at Los Angeles, where he is currently working toward the Ph.D. degree.

His research interests include computer-aided design for programmable fabrics, wireless health, and brain-computer interface.

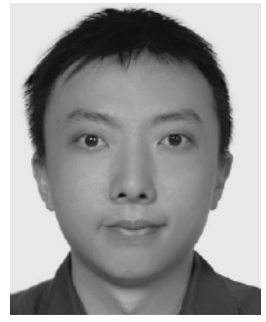

Jueh-Yu Lee (S'08) received the B.S. degree in computer science from National Chiao-Tung University, Hsinchu, Taiwan, in 2004, and the M.S. degree in electrical engineering from National Taiwan University, Taipei, Taiwan, in 2006. He is currently working toward the Ph.D. degree in the Electrical Engineering Department, University of California, Los Angeles (UCLA).

His current research interests include computeraided design for field-programmable gate array synphysical synthesis, and wireless health. thesis and application-specified integrated circuit

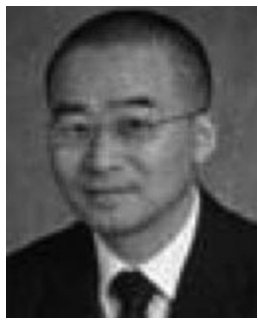

Lei He (M'99-SM'08) received the Ph.D. degree in computer science from University of California, Los Angeles (UCLA) in 1999.

$\mathrm{He}$ is a Professor at the Electrical Engineering Department, University of California, Los Angeles (UCLA) and was a Faculty Member at the University of Wisconsin, MA, between 1999 and 2002. He also held visiting or consulting positions with Cadence, Empyrean Soft, Hewlett-Package, Intel, and Synopsys, and was Technical Advisory Board Member for Apache Design Solutions and Rio Design Automation. His research interests include modeling and simulation, VLSI circuits and systems, and cyber physical systems. He has published a book and more than 200 technical papers with 12 best paper nominations mainly from Design Automation Conference and International Conference on Computer-Aided Design.

Prof. He is a recipient of five Best Paper or Best Contribution awards including the ACM Transactions on Electronic System Design Automation 2010 Best Paper Award.

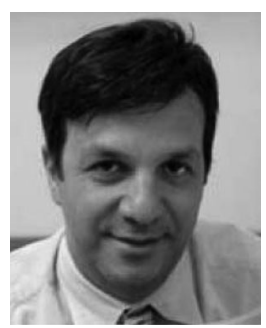

Majid Sarrafzadeh (F'96) received the Ph.D. degree, in 1987, from the University of Illinois at Urbana-Champaign in electrical and computer engineering.

He joined Northwestern University as an Assistant Professor, in 1987. In 2000, he joined the Computer Science Department at the University of California at Los Angeles (UCLA). He is currently Co-Director of the UCLA Wireless Health Institute where he has a few dozen active projects with medical doctors and nurses around the world. His recent research interests lie in the area of embedded and reconfigurable computing with emphasis on healthcare. He has published approximately 370 papers, coauthored 5 books, and is a named inventor on many US patents. He has collaborated with many industries in the past 25 years industries and was the architect of Monterey Design Systems. He was a cofounder of Hier Design, Inc. He has recently cofounded Medisens and BioAssyst; both companies in the area of Wireless Health.

Dr. Sarrafzadeh has served on the technical program committee of numerous conferences and has been a general chair of many of them. 